\title{
CDISC SDTM Sudden Death Syndrome Type Terminology
}

National Cancer Institute

\section{Source}

National Cancer Institute. CDISC SDTM Sudden Death Syndrome Type Terminology. NCI Thesaurus. Code C101852.

Terminology associated with the sudden death syndrome type codelist of the Clinical Data Interchange Standards Consortium (CDISC) Study Data Tabulation Model (SDT M). 